\title{
Calcium-dependent modulation and plasma membrane targeting of the AKT2 potassium channel by the CBL4/ CIPK6 calcium sensor/protein kinase complex
}

\author{
Katrin Held ${ }^{1, *}$, François Pascaud ${ }^{2, *}$, Christian Eckert ${ }^{1}$, Pawel Gajdanowicz ${ }^{3}$, Kenji Hashimoto ${ }^{1}$ \\ Claire Corratgé-Faillie ${ }^{2}$, Jan Niklas Offenborn ${ }^{1}$, Benoît Lacombe ${ }^{2}$, Ingo Dreyer ${ }^{3}$, Jean-Baptiste Thibaud ${ }^{2}$, \\ Jörg Kudla ${ }^{1}$
}

${ }^{1}$ Institut für Biologie und Biotechnologie der Pflanzen, Universität Münster, 48149 Münster, Germany; ${ }^{2}$ Biochimie et Physiologie Moléculaire des Plantes (UMR 5004 CNRS-INRA-SupAgro-UM2), Campus INRA-SupAgro, Place Viala, F34060 Montpellier Cedex 1, France; ${ }^{3}$ Institut für Biochemie und Biologie, Universität Potsdam, Karl-Liebkencht-Str. 24/25, 14476 Potsdam-Golm, Germany

Potassium $\left(\mathrm{K}^{+}\right)$channel function is fundamental to many physiological processes. However, components and mechanisms regulating the activity of plant $\mathrm{K}^{+}$channels remain poorly understood. Here, we show that the calcium $\left(\mathrm{Ca}^{2+}\right)$ sensor CBL4 together with the interacting protein kinase CIPK6 modulates the activity and plasma membrane (PM) targeting of the $\mathrm{K}^{+}$channel AKT2 from Arabidopsis thaliana by mediating translocation of AKT2 to the PM in plant cells and enhancing AKT2 activity in oocytes. Accordingly, akt2, cbl4 and cipk6 mutants share similar developmental and delayed flowering phenotypes. Moreover, the isolated regulatory C-terminal domain of CIPK6 is sufficient for mediating CBL4- and $\mathrm{Ca}^{2+}$-dependent channel translocation from the endoplasmic reticulum membrane to the PM by a novel targeting pathway that is dependent on dual lipid modifications of CBL4 by myristoylation and palmitoylation. Thus, we describe a critical mechanism of ion-channel regulation where a $\mathrm{Ca}^{2+}$ sensor modulates $\mathrm{K}^{+}$channel activity by promoting a kinase interaction-dependent but phosphorylation-independent translocation of the channel to the PM.

Keywords: calcium sensor; protein kinase; potassium channel; signal transduction

Cell Research (2011) 21:1116-1130. doi:10.1038/cr.2011.50; published online 29 March 2011

\section{Introduction}

Potassium $\left(\mathrm{K}^{+}\right)$is an essential nutrient for plants. To efficiently acquire $\mathrm{K}^{+}$despite its fluctuating availability in the soil and to adjust $\mathrm{K}^{+}$distribution within the plant to variable environmental conditions and developmental requirements a tight regulation of $\mathrm{K}^{+}$-transporting proteins is fundamental. Recent studies have identified a regulatory circuit in which the calcineurin $\underline{B}$-like proteins,

\footnotetext{
*These two authors contributed equally to this work. Correspondence: Jean-Baptiste Thibaud ${ }^{\mathrm{a}}$, Jörg Kudla ${ }^{\mathrm{b}}$

${ }^{\mathrm{a}}$ Tel: +33-499-612609; Fax: +33-675-25737

E-mail: thibaud@supagro.inra.fr

${ }^{\mathrm{b}}$ Tel: +49-251-8324813; Fax: +49-251-8323311

E-mail: jkudla@uni-muenster.de

Received 27 July 2010; revised 30 November 2010; accepted 10 December 2010; published online 29 March 2011
}

CBL1 and CBL9, specifically interact with and activate the $\mathrm{CBL}$-interacting protein kinase CIPK23 to phosphorylate and positively modulate the activity of the inwardrectifying $\mathrm{K}^{+}$channel AKT1 [1, 2]. CBL1(CBL9)CIPK23-mediated phosphorylation of AKT1 is essential for transport activity of this channel in oocytes and for proper growth of plants under low- $\mathrm{K}^{+}$conditions [1-3]. These studies directly linked ion-channel regulation to a complex $\mathrm{Ca}^{2+}$-decoding network that is formed by 10 CBL calcium $\left(\mathrm{Ca}^{2+}\right)$ sensor proteins and 26 CIPKs in Arabidopsis thaliana $[4,5]$. Specific complex formation of defined CBL proteins with distinct subsets of CIPKs has been shown to be important for generating signaling specificity in this $\mathrm{Ca}^{2+}$-decoding network [6-8]. CBL proteins, which are most similar to calcineurin $\mathrm{B}$ and neuronal $\mathrm{Ca}^{2+}$ sensor proteins, and their interacting CIPKs have been recognized as fulfilling critical functions in diverse $\mathrm{Ca}^{2+}$-dependent processes in plants and several mu- 
tant studies have unraveled the importance of single CBL and CIPK proteins for proper abiotic stress responses of plants [8-11]. Of the $10 \mathrm{CBL}$ proteins, 4 harbor potential $\mathrm{N}$-terminal myristoylation motifs that could promote the membrane association of these proteins. For CBL1, it has been shown that dual lipid modifications by myristoylation and palmitoylation (the latter is also referred to as S-acylation) are required for its function and for localization of this $\mathrm{Ca}^{2+}$ sensor at the plasma membrane (PM). This localization is achieved by a two-step targeting process in which initial myristoylation results in localization at the endoplasmic reticulum (ER), and subsequent palmitoylation is crucial for ER-to-PM trafficking [12]. Moreover, the lipid-modification status of the $\mathrm{Ca}^{2+}$ sensor moiety determines the cellular localization of preassembled CBL/CIPK complexes and thereby dual lipid modifications of PM-targeted CBL proteins may represent an essential prerequisite for CBL-CIPK-mediated regulation of PM-located ion transporters and channels.

$\mathrm{Up}$ to now it has remained unknown whether CBL/ CIPK complexes regulate any other $\mathrm{K}^{+}$channels than AKT1 in plants. Among the nine Shaker-type $\mathrm{K}^{+}$channel subunits expressed in A. thaliana, AKT2 is unique because it exhibits weak inward-rectifying activity in oocytes $[13,14]$, and several studies have suggested regulation of this channel by unknown protein kinases and the protein phosphatase PP2CA [15-18]. We were therefore especially interested in further understanding the molecular mechanisms modulating AKT2 activity. Here, we report the identification of the CBL4/CIPK6 $\mathrm{Ca}^{2+}$ sensor/protein kinase complex as a critical component for modulation of AKT2 activity. We show that CBL4 $\mathrm{Ca}^{2+}$-dependently mediates efficient translocation of AKT2 from the ER membrane to the PM by a targeting pathway that relies on dual lipid modifications of CBL4 by myristoylation and palmitoylation. The importance of CBL4 and CIPK6 function for proper AKT2 regulation is underscored by the fact that loss of either CBL4 or CIPK6 function in respective mutant lines leads to the same developmental phenotypes as complete loss of AKT2 function. Our cell biological analyses in plant tissues and biophysical studies in oocytes provide direct evidence for a kinase-independent role of the regulatory C-terminal domain of CIPK6 in these processes. We suggest that AKT2 is efficiently translocated to the PM by a novel CBL4- and $\mathrm{Ca}^{2+}$-dependent targeting pathway that requires a scaffolding function of CIPK6 and occurs independently of the CIPK6 kinase activity. This supports the idea of multiple pathways for $\mathrm{K}^{+}$channel trafficking. AKT2 modulation by CBL4/CIPK6 complexes could provide a mechanism for $\mathrm{Ca}^{2+}$-dependent trafficking of PM-targeted ion channels that supplements ion channel regulation by protein phosphorylation.

\section{Results}

CBL4 association with CIPK6 specifically facilitates $\mathrm{Ca}^{2+}$-dependent activation of the AKT2 $\mathrm{K}^{+}$channel

To identify CBL-interacting protein kinases that potentially interact with AKT2, we performed yeast twohybrid analyses that combined all 26 known CIPKs from A. thaliana with a C-terminal cytoplasmic fragment of AKT2 encompassing amino acids 324-802. This approach identified CIPK6 as a kinase specifically interacting with AKT2 (Figure 1A). Subsequently, using yeast two-hybrid assays we analyzed the PM-localized CBL proteins that can interact with CIPK6. These experiments revealed interaction of CIPK6 with CBL1, CBL4 and CBL9 but indicated no interaction with the closely related $\mathrm{Ca}^{2+}$ sensor, $\mathrm{CBL} 5$ (Figure 1B). Specific interaction of CIPK6 but not of CIPK23 with full-length AKT2 was further confirmed by in planta BiFC (bimolecular fluorescence complementation) experiments in transiently transformed Nicotiana benthamiana leaves (Figure 1C). The YFP fluorescence formed by the assembly of AKT2YC and YN-CIPK6 accumulated predominantly at ERrich regions of the cell (Supplementary information, Figure S1A). These results are in line with the observation that many characterized channel subunits are retained at the ER and require association with escort proteins, protein modification or oligomerization for trafficking beyond the ER [19-21] and confirm previous AKT2 1ocalization studies [22].

To delineate the functional significance of the observed CBL-CIPK6 and CIPK6-AKT2 interactions, we expressed different combinations of cRNAs in oocytes and recorded the resulting currents in two-electrode voltage-clamp (TEVC) experiments (Figure 1E-1H). A typical voltage protocol and typical traces for these measurements are depicted in Figure 1D-1F. Expression of AKT2 alone resulted in basal $\mathrm{K}^{+}$currents that reflect the activity of AKT2 on its own $(100 \%$ current at -155 $\mathrm{mV}$, Figure $1 \mathrm{H}$ ) and expression of AKT2 in combination with either CBL4 or CIPK6 did not result in significant enhancement of currents (max. 150\%). However, coexpression of AKT2 with CBL4 and CIPK6 dramatically increased the recorded currents to $380 \%$ (Figure $1 \mathrm{H}$ ). Although CBL1 and CBL9 both interacted with CIPK6 (Figure 1B), co-expression of either of these $\mathrm{Ca}^{2+}$ sensors with CIPK6 and AKT2 did not lead to a significant increase in channel activity (Figure $1 \mathrm{H}$ ). Co-expression of AKT2 with CBL1 and CIPK23, that have been shown to activate AKT1 [1] (Figure 1I), did not significantly change AKT2 activity (Figure 1H). Similarly, CBL4 
A

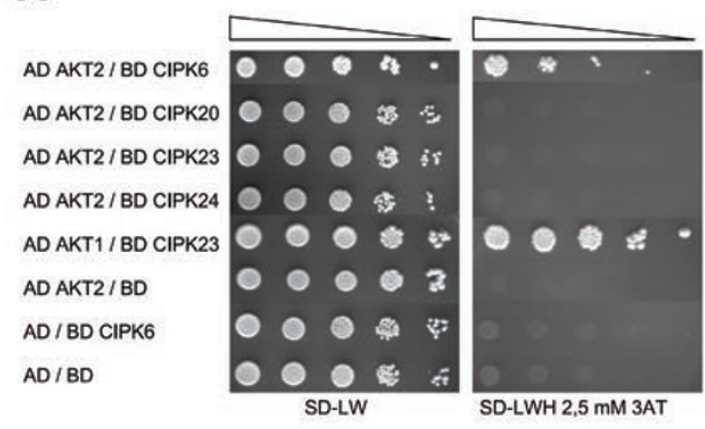

C

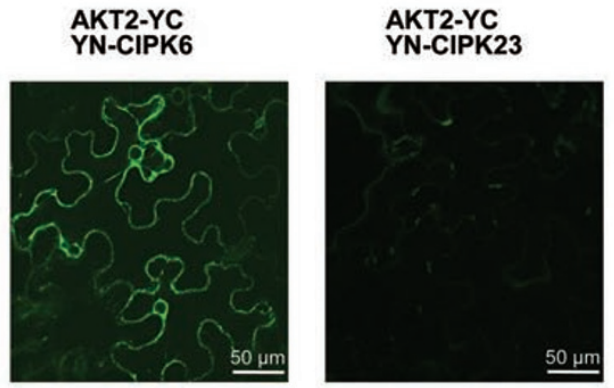

H

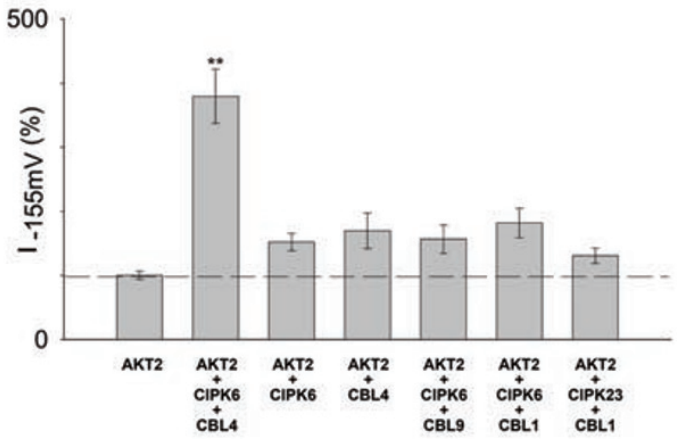

J

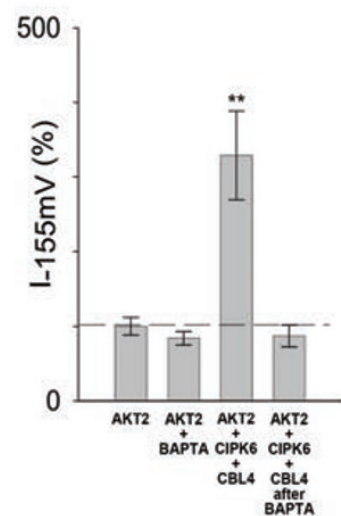

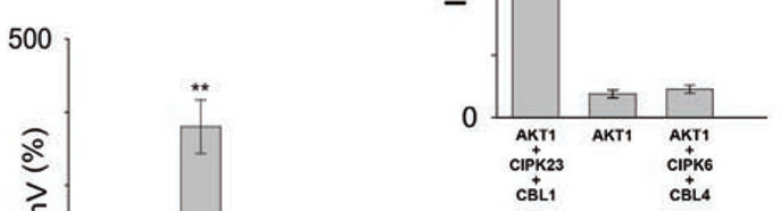

I 120

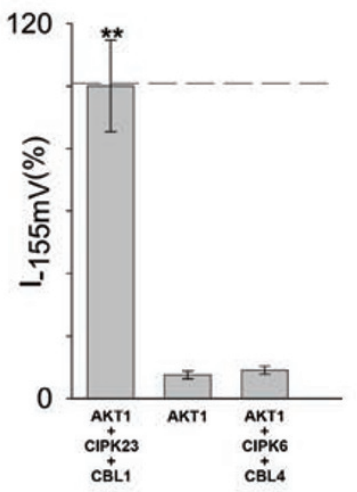

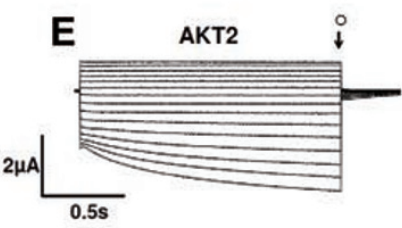

$\mathbf{F}$

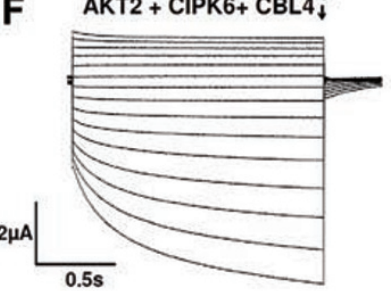

G
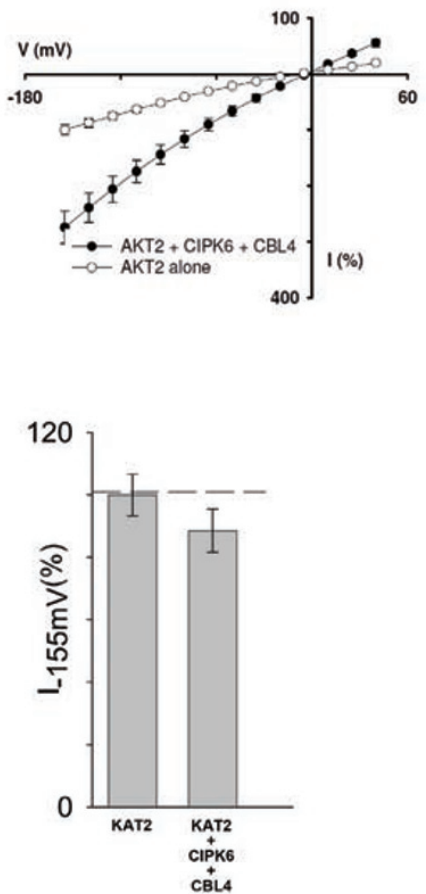
and CIPK6 did not activate AKT1, thereby indicating the specificity of the observed AKT2 regulation (Figure 1I, Supplementary information, Figure S1B). Also, coinjection of CBL4 and CIPK6 did not modulate the activity of the related shaker-type $\mathrm{K}^{+}$channel, KAT2 (Figure 1I, Supplementary information, Figure S1C), thereby further corroborating the functional specificity of the observed AKT2 activation. To address the $\mathrm{Ca}^{2+}$ dependence of AKT2 activation, TEVC recordings were performed either after BAPTA injection in oocytes or by replacing wild-type CBL4 with a mutated CBL4 version, harboring a deletion of three amino acids in the third EF hand (mutant allele sos 3-1, here designated as CBL4 $\triangle \mathrm{EF}$ ) that has been previously shown to impair CBL4 $\mathrm{Ca}^{2+}$ binding [23]. Both experimental designs congruently abolished CBL4-CIPK6-mediated activation of AKT2, thereby unequivocally demonstrating the strict $\mathrm{Ca}^{2+}$ dependence of AKT2 activation (Figure 1J). Taken together these results provide strong support for the conclusion that CBL4 together with CIPK6 specifically and $\mathrm{Ca}^{2+}$-dependently modulates the $\mathrm{K}^{+}$channel activity of AKT2.
To dissect the mechanisms by which CBL4 affects AKT2 activity we next investigated whether CBL4 and CIPK6 affect the voltage-gating modes of the cellular AKT2 population. Previous studies have shown that, depending on their phosphorylation status, AKT2 channels display either $\mathrm{K}^{+}$-selective inward-rectifying (gating mode\#1) or $\mathrm{K}^{+}$-selective "open leak" (gating mode\#2) features [17]. However, comparative TEVC analyses of oocytes expressing either AKT2 alone or AKT2 in combination with CBL4 and CIPK6 did not indicate any difference in the proportion of time-dependent (gating mode\#1) and instantaneous (gating mode\#2) (Supplementary information, Figure S2A) currents and did not reveal detectable differences in the voltage gating of the time-dependent fraction of the AKT2 current (Supplementary information, Figure S2B). In addition, singlechannel AKT2 conductance values determined from cellattached patches of oocytes clamped at $-140 \mathrm{mV}$ were similar in the presence or absence of CBL4 and CIPK6, further excluding any influence of CBL4/CIPK6 on the permeation properties of the open AKT2 channel (Sup-

Figure 1 CIPK6 specifically interacts with the AKT2 C-terminus, and CBL4/CIPK6 complexes Ca ${ }^{2+}$-dependently modulate AKT2 activity. (A) Yeast two-hybrid analysis of CIPK-AKT2 interaction. The yeast strain PJ69-4A containing the indicated plasmid combinations was grown on the indicated media for 14 days at $23{ }^{\circ} \mathrm{C}$. The published interaction of AD-AKT1 with BD-CIPK23 served as positive control. Decreasing cell densities in the yeast dilution series are illustrated by narrowing triangles. (B) Yeast two-hybrid analysis of CIPK6-CBL interaction. The yeast strain PJ69-4A containing the indicated plasmid combinations was grown on the indicated media for 7 days at $23^{\circ} \mathrm{C}$. The published interaction of AD-CIPK1 with BD-CBL1 served as positive control. (C) CIPK6 interacts with AKT2 in planta. Microscopic analysis of BiFC complexes formed by the indicated plasmid combinations after 4 days of infiltration in $N$. benthamiana leaves. (D-G) Increase of AKT2 currents upon co-expression with CIPK6 and CBL4. (D) Typical voltage clamp protocol. Voltage-clamp pulses (1.6 s) from $+40 \mathrm{mV}$ to -170 $\mathrm{mV}$ (step: $-15 \mathrm{mV}$, holding potential: $-40 \mathrm{mV}$ ). ( $\mathbf{E}$ and $\mathbf{F})$ Typical current traces elicited by the voltage protocol described in panel (D) in oocytes expressing $A K T 2$ or $A K T 2+C I P K 6+C B L 4$. Experiments were performed in a 100 mM $\mathrm{K}^{+}$external solution, currents were recorded 4 days after injection of $A K T 2$ cRNA or AKT2, CIPK6 and CBL4 cRNAs. (G) Current-voltage (I$\mathrm{V})$ curves for oocytes injected with AKT2 cRNA (open symbols) and injected with a mix of AKT2, CIPK6 and CBL4 cRNAs (closed symbols). Current values from recordings like in panels ( $E$ and $F$ ) were normalized to the mean current value at -155 $\mathrm{mV}$ in oocytes injected with $A K T 2 \mathrm{cRNA}$ only. Data are given in percent as mean $\pm \mathrm{SE}$ (symbols cross-referenced to $\mathbf{E}$ and F) with $n=14$ for $A K T 2$ and $n=8$ for $A K T 2+C I P K 6+C B L 4$. $(\mathbf{H})$ Both CIPK6 and CBL4 are required for a Ca ${ }^{2+}$-dependent increase of AKT2 current. Increase of AKT2 current in $X$. laevis oocytes by different CBL/CIPK combinations. Currents recorded in oocytes injected with different mixes of cRNAs were normalized to the mean current value at $-155 \mathrm{mV}$ in oocytes injected with $A K T 2$ cRNA only. Data are displayed as mean \pm SE for the following injected mixes of $\mathrm{cRNAs}$ : $A K T 2$ ( $n=48)$, $A K T 2+C I P K 6+C B L 4(n=44), A K T 2+C I P K 6(n=16), A K T 2+C B L 4(n=15), A K T 2+C I P K 6+C B L 9(n=9), A K T 2+C I P K 6+C B L 1$ $(n=9), A K T 2+C I P K 23+C B L 1(n=6)$. (I) Both AKT1 and KAT2 are insensitive to CIPK6/CBL4. (Left) Currents in oocytes injected with AKT1 CRNA and either CIPK23 and CBL1 or CIPK6 and CBL4 CRNAs. Data are current values at $-155 \mathrm{mV}$ normalized to the mean current value at $-155 \mathrm{mV}$ in oocytes injected with $A K T 1, C I P K 23$ and $C B L 1 \mathrm{cRNAs}$ (mean \pm SE, $n=5,7$ and 6 for $A K T 1+C I P K 23+C B L 1, A K T 1$ and $A K T 1+C I P K 6+C B L 4$, respectively). (Right) Currents in oocytes injected with either KAT2 cRNA or a mix of KAT2, CIPK6 and CBL4 cRNAs. Data are current values at $-155 \mathrm{mV}$ normalized to the mean current value at $-155 \mathrm{mV}$ in oocytes injected with $K A T 2 \mathrm{cRNA}$ (mean $\pm \mathrm{SE}, n=7$ ). (J) $\mathrm{Ca}^{2+}$ dependence of AKT2 current modulation by CIPK6/CBL4. (Left) Currents in oocytes injected with either AKT2 cRNA or a mix of AKT2, CIPK6 and CBL4 cRNAs were recorded before and $5 \mathrm{~min}$ after a $50 \mathrm{mM}$ BAPTA injection. Data are current values at $-155 \mathrm{mV}$ normalized to the mean current value at $-155 \mathrm{mV}$ in oocytes injected with $A K T 2 \mathrm{cRNA}$ only (mean $\pm S E, n=10$ ). (Right) CIPK6/CBL4 stimulation of AKT2 current is abolished by an EF hand mutation in CBL4. Oocytes were injected with different combinations of cRNAs: AKT2, $A K T 2+C I P K 6+C B L 4$ or $A K T 2+C I P K 6+C B L 4 \triangle E F$. Data are current values at $-155 \mathrm{mV}$ normalized to the mean current value at $-155 \mathrm{mV}$ in oocytes injected with $A K T 2$ CRNA only (mean \pm SE, $n=10)$. ${ }^{* *}$ Results with significant difference in values $(\mathbf{H}-J)$. 


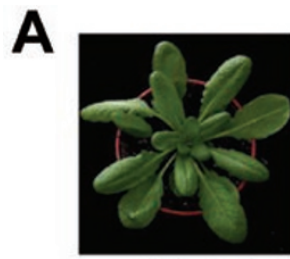

WT-Ws
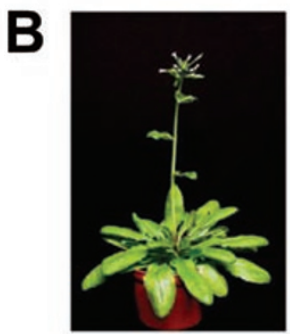

C

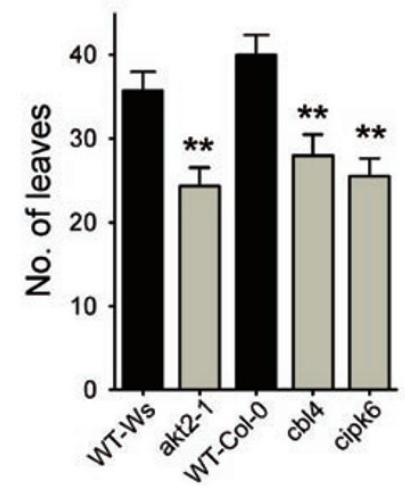

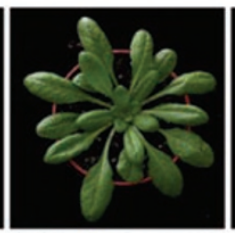

WT-Col-0
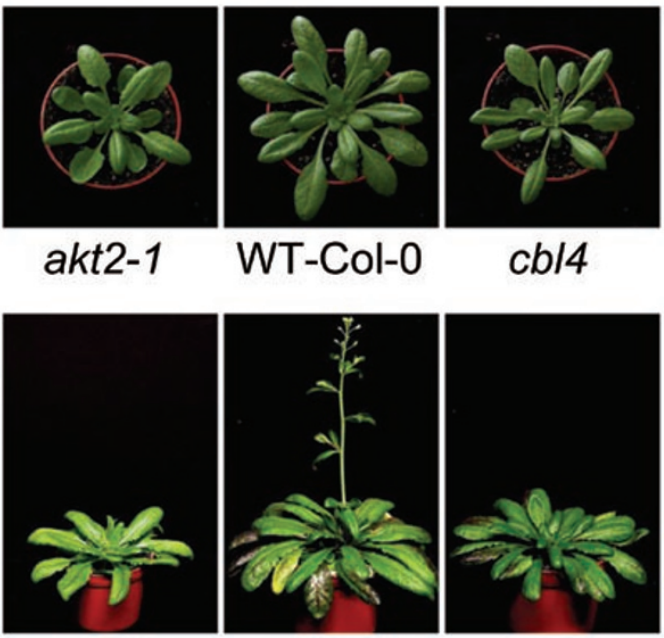

cb/4
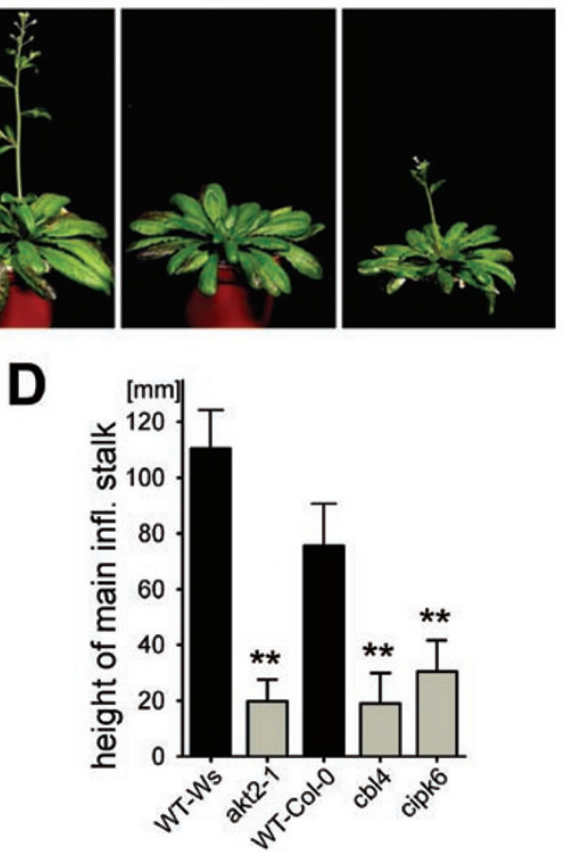

Figure 2 Loss-of-function: akt2-1, cb/4 and cipk6 mutant plants exhibit similar developmental and flowering phenotypes in short day conditions. Plants were grown in a 12 h day/12 h night cycle. (A) Plant development 6 weeks after sowing (WAS). (B) Plants 8 WAS (WT-Ws and akt2-1) and 9 WAS (WT-Col-0, cb/4 and cipk6). (C) Number of leaves determined 6 WAS ( $n=20-$ 24). (D) Height of the main inflorescence stalk determined 8 WAS (WT-Ws and akt2-1; $n=23$ in each case) and 9 WAS (WTCol-0, cb/4 and cipk6; $n=22-23$ ). Data in panels $(\mathbf{C}$ and $\mathbf{D})$ are depicted as mean $\pm S D$. ** Results with significant difference in values.

plementary information, Figure S2C). These data exclude the possibility that CBL4/CIPK6 modulate AKT2 activity by affecting the voltage gating of this channel.

Loss of either CBL4 or CIPK6 function causes the same developmental phenotypes as the loss of AKT2 function

We investigated whether CBL4, CIPK6 and AKT2 function may be similarly required for distinct physiological processes in plants by comparatively characterizing a published T-DNA-induced mutant allele of AKT2 (akt2-1) [24, 25] with newly isolated mutant alleles of CBL4 and CIPK6 (GABI_015F02: designated cbl4 and GABI_448C12: designated cipk6, respectively; Supplementary information, Figure S3A and S3B). Previous studies reported delayed rosette development and flowering of the akt2-1 mutant in comparison with wild type as a consequence of impaired sugar loading and long-dis- tance transport due to the lack of AKT2 function, which is critical for $\mathrm{K}^{+}$-dependent repolarization of phloem cells that is itself essential for regulating sucrose $/ \mathrm{H}^{+}$symporters that drive sugar transport [25]. We did not observe significant differences of plant developmental parameters when mutant and corresponding wild-type plants (Ws for akt2-1 and Col-0 for cbl4, cipk6) were cultivated in long day conditions. However, when grown in short days the development of all mutant lines was delayed in comparison with the respective wild types as exemplified by a reduced rosette size and later flowering (Figure $2 \mathrm{~A}$ and $2 \mathrm{~B}$ ). Quantitative evaluation of the leaf number revealed the development of about $30 \%$ less leaves in akt2-1, cbl4 and cipk6 mutants than in the wild type after 6 weeks of cultivation (Figure 2C). The development of the main inflorescence stalk was also delayed in all mutant lines. While after 54 days of cultivation Ws wild 


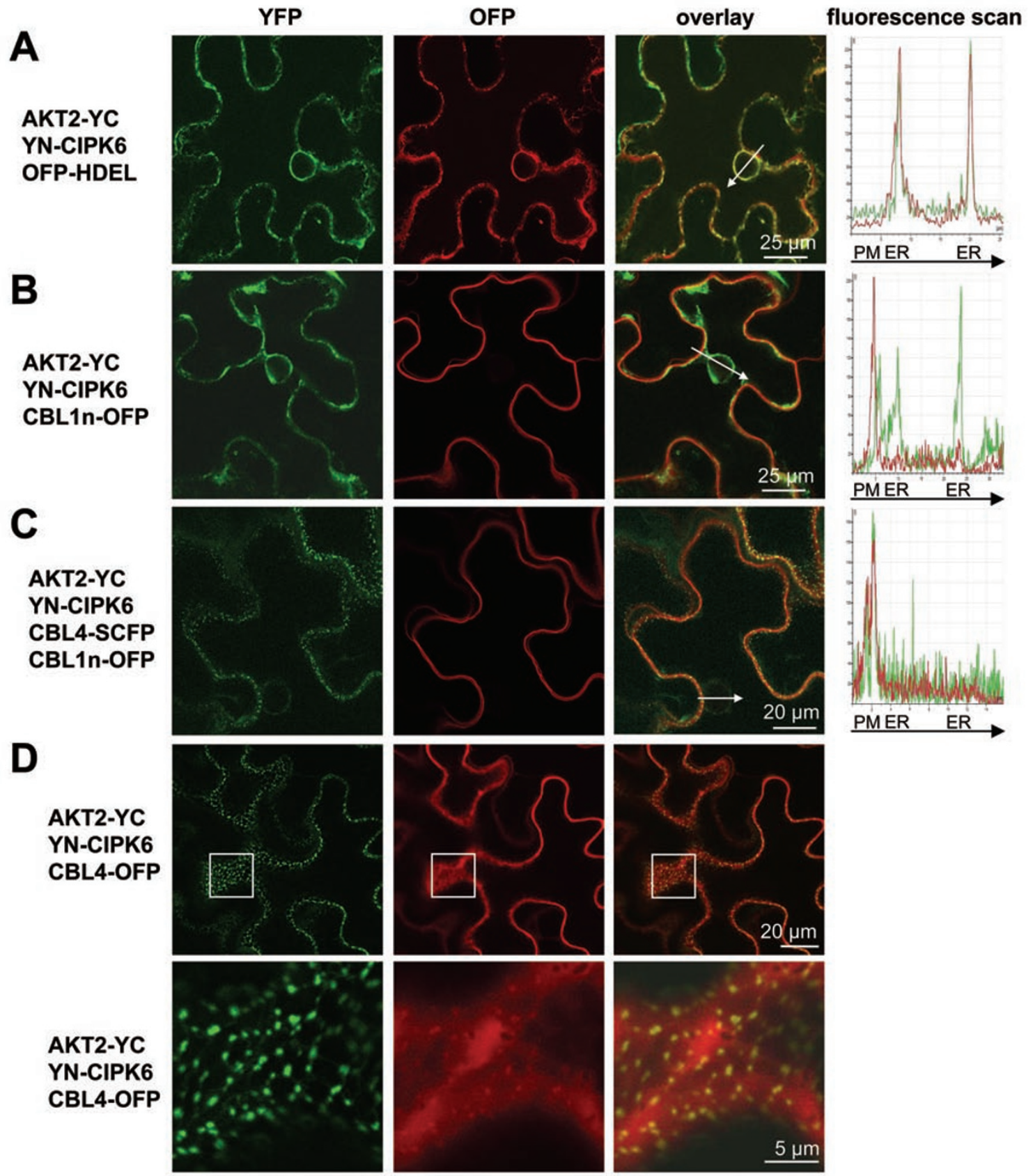

Figure 3 CBL4-dependent ER-to-PM translocation of AKT2. (A-D) Microscopic analysis of the median cellular plane of N. benthamiana epidermal cells transiently expressing the plasmid combinations indicated at the left. (A) Formation and ERlocalization of AKT2/CIPK6 (green) complexes as revealed by BiFC and co-localization with the ER-marker OFP-HDEL (red). Arrows mark the region and direction in which the distribution of fluorescence intensities was determined. (B) The localization of AKT2/CIPK6 complexes (green) is distinct from PM-marker CBL1n-OFP (red). (C and D) Localization of AKT2/CIPK6 BiFC complexes after co-expression of CBL4-OFP or CBL4-SCFP is shifted toward the PM. (C) Co-localization of AKT2/CIPK6 complexes after co-expression with CBL4-SCFP and the PM-marker CBL1n-OFP reveal PM-localization of AKT2/CIPK6 in the presence of CBL4 and a dramatic reduction of the AKT2-CIPK6-indicating fluorescence signal in the perinuclear envelope is detected by a fluorescence scan. A white arrow marks the region and direction in which the distribution of fluorescence intensities was determined. (Further details and the distribution of CBL4-SCFP fluorescence are shown in Supplementary information, Figure S4B.) (D) View into the optical plane of the PM indicating CBL4-dependent accumulation of AKT2/CIPK6 BiFC complexes in punctate structures of the PM. Bottom panel: enlargement of the area indicated by a white frame in the top panel. 

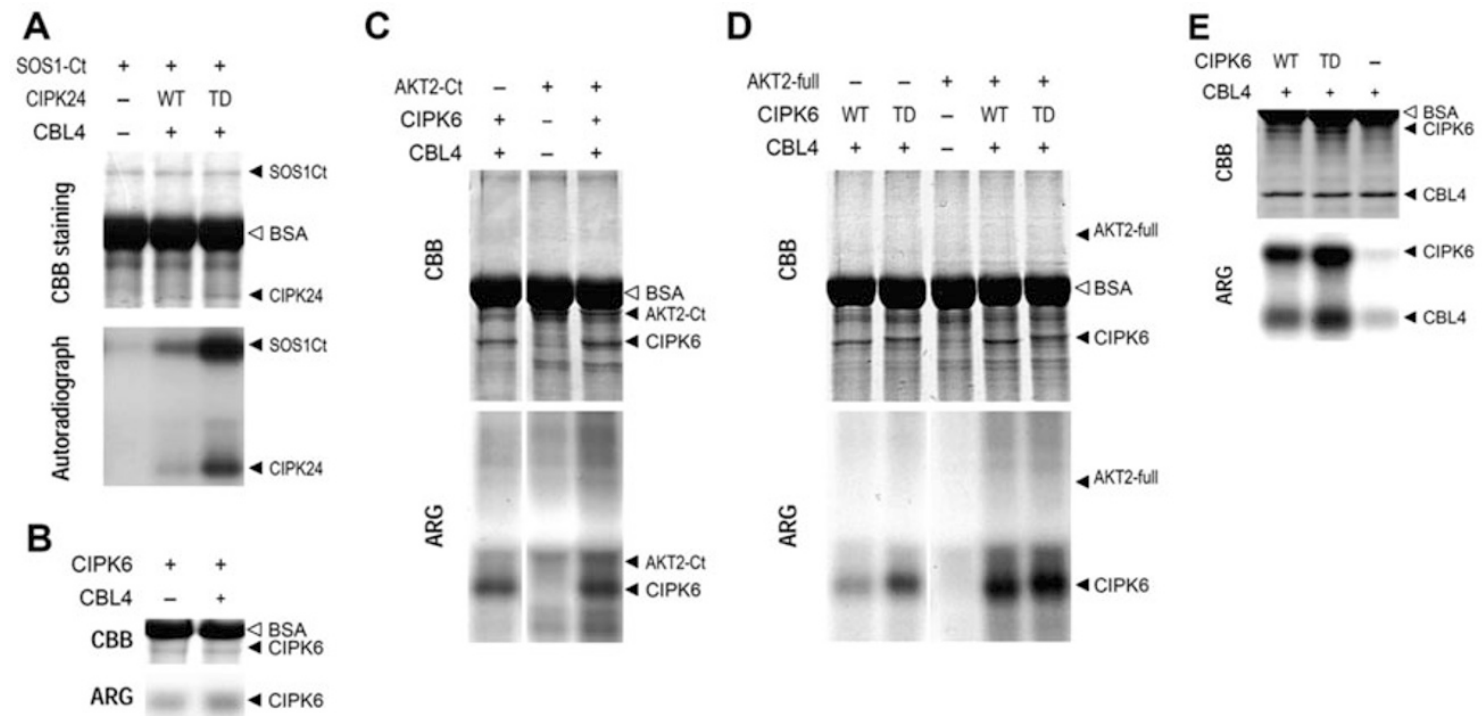

Figure 4 In vitro phosphorylation assays detect no phosphorylation of AKT2 by CIPK6. (A-E) Upper panels depict Coomassie Brilliant Blue stainings (CBB) of recombinant proteins and lower panels present the corresponding auto-radiographs (ARG) after phosphorylation assays. (A) The suitability of the experimental conditions was verified by CBL4-CIPK24-mediated phosphorylation of the SOS1 C-terminus (SOS1-Ct). A hyperactive mutant, CIPK24T168D (indicated as TD), exhibited enhanced kinase activity. (B) CIPK6 displayed auto-phosphorylation activity, which was influenced by CBL4. (C and D) Phosphorylation of the C-terminal fragment or full-length protein of AKT2 by the CBL4/CIPK6 complex was not detectable. (E) CIPK6 efficiently trans-phosphorylated CBL4.

type plants displayed an average inflorescence stalk height of about $110 \mathrm{~mm}$, a stalk of only $20 \mathrm{~mm}$ was detectable in akt2-1 plants. Similarly, after 62 days cbl4 and cipk6 mutants exhibited an inflorescence stalk height of 20 and $30 \mathrm{~mm}$, respectively, compared with $75 \mathrm{~mm}$ in the Col0 wild type (Figure 2D). Importantly, these phenotypes were fully complemented to wild-type values when akt21, cbl4 and cipk6 mutants were transformed with the respective wild-type cDNAs, thereby causally linking the observed phenotypes to the loss of either CBL4, CIPK6 or AKT2 function (Supplementary information, Figure S3C, S3D). These results support the notion that similar developmental phenotypes of cbl4, cipk6 and akt2-1 mutant lines coincide with the observed direct functional interactions between these proteins. Importantly, the fact that the loss of either CBL4 or CIPK6 function impedes plant development and flowering similarly as the complete loss of AKT2 function underscores the importance of the regulatory function of the CBL4/CIPK6 complex for proper AKT2 function in plants.

Upon interaction with CIPK6, CBL4 mediates ER-to-PM translocation of AKT2

In order to gain further insights into the mode of CBL4-CIPK6-mediated AKT2 regulation, we evaluated whether CBL4 may influence the sub-cellular distribution of AKT2 in plant cells. To this end, we transiently expressed BiFC constructs of AKT2-YC and YN-CIPK6 together with different combinations of sub-cellular marker proteins and with or without the co-expression of CBL4SCFP or CBL4-OFP fusion proteins in $N$. benthamiana leaves, and we determined the resulting fluorescence pattern indicating the localization of AKT2 (Figure 3). The netted pattern of BiFC fluorescence, and especially the accumulation of fluorescence at membranes around the nucleus, was suggestive of an ER accumulation of AKT2 and CIPK6. Indeed, co-expression of AKT2-YC and YN-CIPK6 with the ER-marker protein, OFP-HDEL [12], revealed a predominant localization of the CIPK6/ AKT2 complex at the ER as evidenced by fluorescence overlay analysis and fluorescence intensity scans of the observed green (YFP) and red (OFP) fluorescence patterns (Figure 3A). In these fluorescence intensity scans OFP-HDEL clearly co-localized with BiFC fluorescence emitted upon interaction of AKT2 with CIPK6 (Figure $3 \mathrm{~A})$. Co-expression of the PM-marker protein, CBL1nOFP, in which the first $12 \mathrm{~N}$-terminal amino acids of CBL1 are fused to OFP [12], with CIPK6 and AKT2 further confirmed the only minor co-localization with the PM-marker protein (Figure 3B, the separation of the ERand PM-marker fluorescence of OFP-HDEL and CBL1nGFP by intensity scans is demonstrated in Supplementary 
A

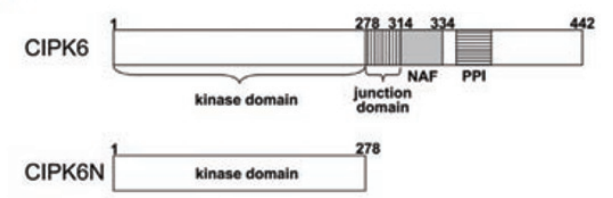

CIPK6C
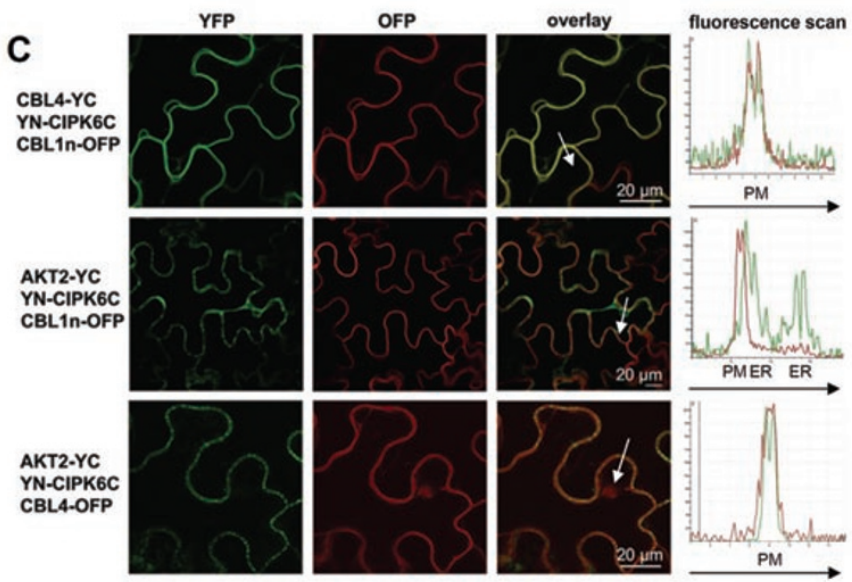

B

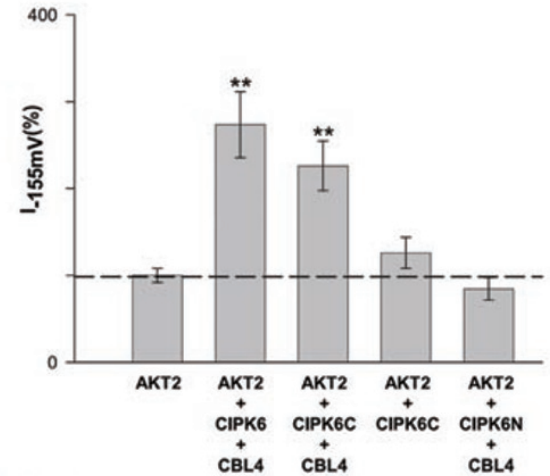

D

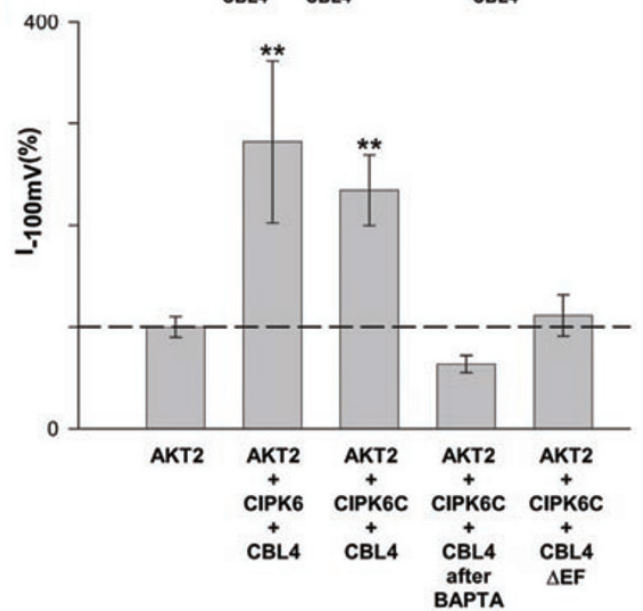

E

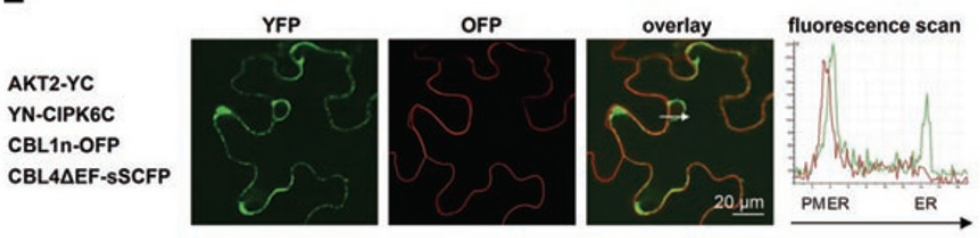

Figure 5 Phosphorylation-independent $\mathrm{Ca}^{2+}$-dependent modulation of AKT2 by CBL4/CIPK6. (A) Schematic presentation of CIPK6 and the CIPK6N and CIPK6C constructs generated in this study. NAF: NAF domain mediating CBL interaction and PPI: phosphatase interaction domain. (B) CBL4 in combination with CIPK6C phosphorylation-independently activates AKT2. Currents recorded in oocytes injected with different mixes of cRNAs were normalized to the mean current value at $-155 \mathrm{mV}$ in oocytes injected with $A K T 2$ cRNA only. Data are representative of three independent experiments and are displayed as mean $\pm S E(n=$ number of tested oocytes) for the following injected mixes of cRNAs: AKT2 $(n=15), A K T 2+C I P K 6+C B L 4(n$ = 9), AKT2+CIPK6C+CBL4 $(n=11), A K T 2+C I P K 6 C(n=10), A K T 2+C I P K 6 N+C B L 4(n=9)$. (C) Microscopic analysis of the median cellular plane of $N$. benthamiana epidermal cells transiently expressing the plasmid combinations indicated at the left. Top panel: BiFC analysis reveals the interaction of CBL4 with the kinase domain deletion construct of CIPK6 (CIPK6C) predominantly at the PM. Middle panel: CIPK6C still interacts with AKT2 despite the absence of the kinase domain. Bottom panel: Co-expression of CBL4-SCFP induces the translocation of AKT2/CIPK6C complexes toward the PM. A white arrow marks the region and direction in which the distribution of fluorescence intensities was determined. (D) $\mathrm{Ca}^{2+}$-dependent stimulation of AKT2 currents by CBL4/CIPK6C. Currents in oocytes injected with AKT2 cRNA, a mix of AKT2, CIPK6C and CBL4 cRNAs or a mix of $A K T 2, C I P K 6 C$ and $C B L 4 \triangle E F$ cRNAs were recorded before and 5 min after a $50 \mathrm{mM}$ BAPTA injection. Data are representative of three independent experiments and are represented as current values at $-100 \mathrm{mV}$ normalized to the mean current value at $-100 \mathrm{mV}$ in oocytes injected with $A K T 2$ cRNA only (mean $\pm \mathrm{SE}, n=6,7,7,6$ and 7 , respectively for $A K T 2, A K T 2+C I P K 6+C B L 4, A K T 2+C I P K 6 C+C B L 4, A K T 2+C I P K 6 C+C B L 4$ after BAPTA and AKT2+CIPK6C+CBL4 $A E F)$. (E) Co-localization of complexes formed by interaction of AKT2-YC with YN-CIPK6C after co-expression with CBL4 $\triangle E F-S C F P$ and the PM-marker CBL1n-OFP reveals the AKT2-CIPK6C-indicating fluorescence signal in ER-enriched cellular region and in the perinuclear envelope as detected by a fluorescence scan. A white arrow marks the region and direction in which the distribution of fluorescence intensities was determined. (Further details and the distribution CBL4 $\triangle E F-S C F P$ fluorescence are shown in Supplementary information, Figure S5B.) 
information, Figure S4A). In cells that simultaneously expressed the BiFC combination AKT2-YC+YN-CIPK6 with CBL4-SCFP and CBL1n-OFP, however, efficient PM translocation of the complex was observed in fluorescence overlay depiction as well as in fluorescence intensity scans (Figure 3C). This transclocation becomes especially evident in the fluorescence scan through the region of the nucleus and the PM (white arrow in Figure 3C), which in contrast to the fluorescence distribution in the scan of Figure 3B depicts strongly reduced green fluorescence in the ER regions and also documents accumulation of the green AKT2/CIPK6 fluorescence at the PM. It is worth to note that cyan fluorescence emitted by CBL4-SCFP was also detected in the cytoplasm and nucleus of these cells, suggesting that only a sub-population of the cellular CBL4-protein pool was involved in translocation of CIPK6/AKT2 to the PM (Supplementary information, Figure S4B). A microscopic analysis focused on the surface of an epidermal cell illustrated the CBL4-dependent accumulation of CIPK6/AKT2 in distinct immobile spotted structures associated with the PM (Figure 3D). This pattern is clearly distinct from the netlike ER structures in which CIPK6/AKT2 BiFC fluorescence is detected in the absence of CBL4 (Supplementary information, Figure S4C). Taken together, our results indicate that CBL4 is required for an efficient ER-to-PM translocation of CIPK6/AKT2 in plant cells that concurs with the observed CBL4-dependent activation of AKT2 by CIPK6 in oocytes (Figure $1 \mathrm{G}$ and $1 \mathrm{H}$ ).

\section{CBL4/CIPK6 complex does not phosphorylate AKT2 in} vitro

We next sought to delineate the potential phosphorylation of AKT2 by CBL4/CIPK6 by in vitro phosphorylation assays. In these experiments we combined recombinant CBL4 and CIPK6 or a hyperactive mutant version of CIPK6 (with a T-to-D exchange in the activation loop of CIPK6, noted CIPK6T182D) with either the Cterminal fragment of AKT2 or full-length AKT2 proteins (Figure 4). Control experiments in which CBL4/CIPK24 efficiently phosphorylated the C-terminus of their target protein, SOS1 [26, 27], verified the suitability of the experimental conditions (Figure 4A). These experiments demonstrated efficient auto-phosphorylation activity of CIPK6 that was slightly modulated by the presence of CBL4 (Figure 4B) but was significantly enhanced as a consequence of the T182D amino acid exchange (Figure 4D). Moreover, CIPK6 as well as CIPK6T182D phosphorylated CBL4 in these assays (Figure 4E), thereby confirming the previously reported phosphorylation of CBL proteins by CIPKs [28] and clearly establishing the trans-phosphorylation ability of the recombinant kinase protein. Nevertheless, we did not obtain any evidence indicating a phosphorylation of AKT2 by CIPK6 despite extensive variations of the tested experimental conditions (Figure 4C and 4D).

The C-terminal regulatory domain of CIPK6 interacts with CBL4 and AKT2, and is sufficient to mediate CBL4and $\mathrm{Ca}^{2+}$-dependent $\mathrm{K}^{+}$channel modulation

These results prompted us to consider a phosphorylation-independent modulation of AKT2 by CIPK6. To distinguish between effects and interactions that solely rely on the kinase domain from kinase-independent functions of CIPK6, we generated two distinct oocyte expression constructs. These constructs harbor either only the N-terminal CIPK6 kinase domain (amino acids 1-278, designated CIPK6N; Figure 5A) or, alternatively a C-terminal fragment of CIPK6 that exclusively contains the NAF domain for $\mathrm{CBL}$ interaction and the regulatory domain of the kinase (amino acids 274-442, designated CIPK6C; Figure 5A). Remarkably, in TEVC analyses of oocytes co-expressing CIPK6C with CBL4 and AKT2, we detected currents of comparable intensity as in cells expressing AKT2 with full-length CIPK6 and CBL4 (Figure 5B). Importantly, such activation of AKT2 was not observed in cells expressing CIPK6N together with CBL4. To exclude that the C-terminal truncation in CIPK6N diminished its kinase activity we performed auto-phosphorylation assays of recombinant CIPK6N (Supplementary information, Figure S6), which clearly revealed the efficient kinase activity of this protein. Moreover, the activation of AKT2 by CIPK6C was strictly dependent on the co-expression of CBL4 (Figure 5B).

Finally, we addressed the functionality of the kinasedeleted version CIPK6C by BiFC analysis in transiently transformed $N$. benthamiana leaves. BiFC complex formation after co-expression of CBL4-YC with YN-CIPK6C confirmed the ability of the kinase-deleted version of CIPK6 to still efficiently interact with the $\mathrm{Ca}^{2+}$ sensor protein (Figure 5C, top panel). While YN-CIPK6C interacted with AKT2-YC in plant cells (Figure 5C, middle panel) a BiFC construct that expressed the kinase domain without the regulatory domain (YN-CIPK6N) was not able to interact with AKT2-YC (Supplementary information, Figure S5A). Fluorescence microscopic analysis of tissues co-expressing AKT2-YC and YN-CIPK6C with CBL4-OFP clearly demonstrated the ability of CBL4 to mediate the ER-to-PM translocation of AKT2/CIPK6C (Figure 5C, bottom panel) as observed before for AKT2/ CIPK6 (Figure 3C). These findings assign the $\mathrm{Ca}^{2+}$ sensor-dependent modulation of AKT2 activity to the Cterminal regulatory domain of CIPK6, which lacks any 

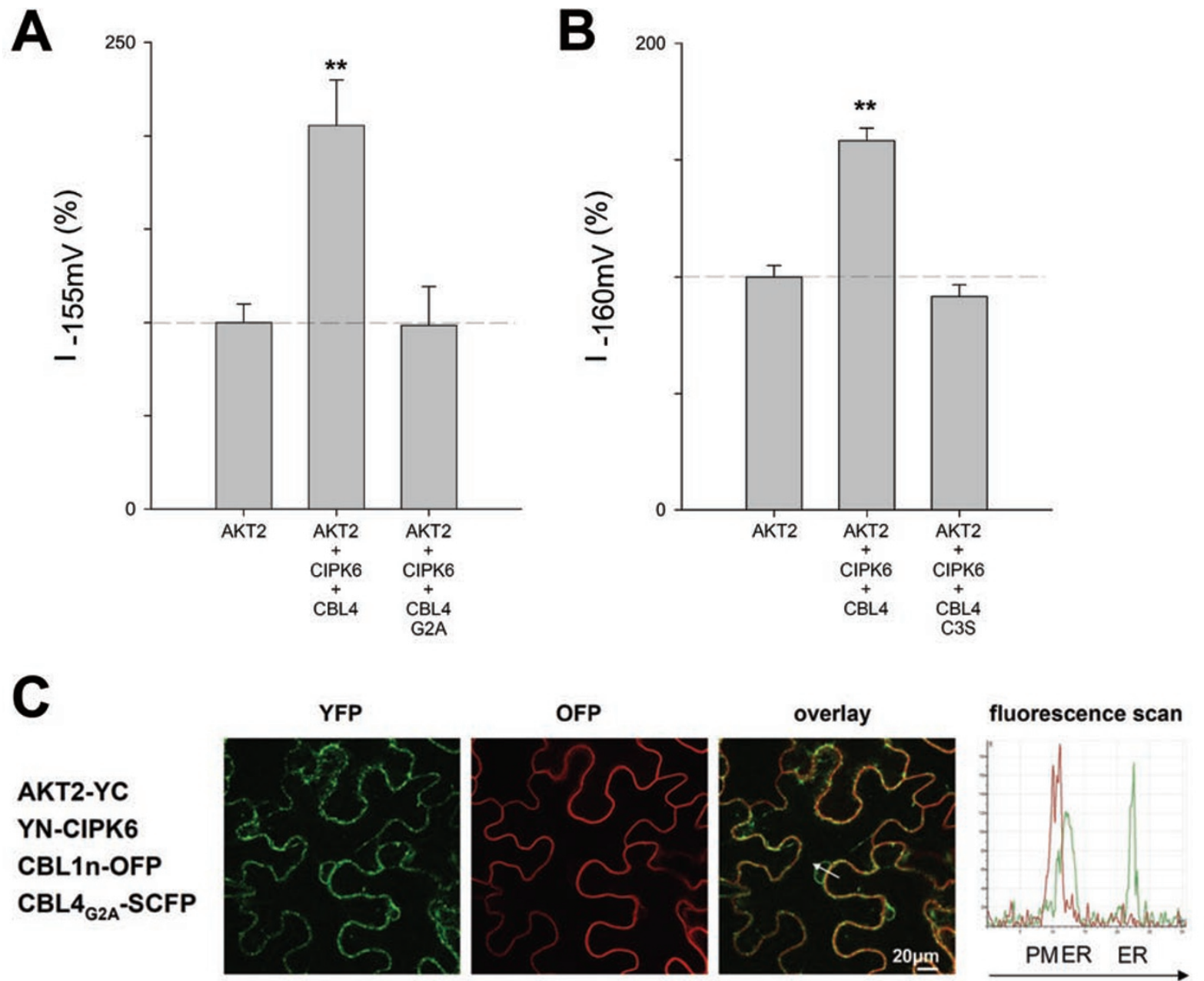

D
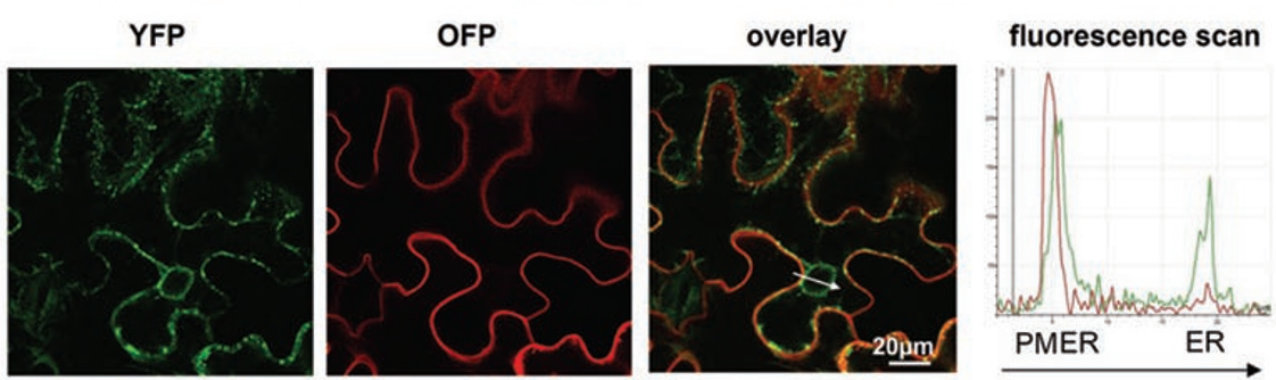

Figure 6 Dual N-terminal lipid modification of CBL4 is necessary for the activation of AKT2 currents by CIPK6 and translocation of AKT2 from the ER to the PM. (A) Activation of AKT2 currents by CIPK6 and CBL4 is impaired if myristoylation of CBL4 is prevented by the CBL4G2A point mutation. Currents recorded in oocytes injected with different mixes of cRNAs were normalized to the mean current value at $-155 \mathrm{mV}$ in oocytes injected with $A K T 2$ cRNA only. Data are displayed as mean \pm SE $(n=$ number of tested oocytes) for the following injected mixes of cRNAs: $A K T 2(n=5), A K T 2+C I P K 6+C B L 4(n=$ $5)$ and $A K T 2+C I P K 6+C B L 4 G 2 A(n=5)$. (B) Dual lipid modification of CBL4 by myristoylation and palmitoylation is required for CBL4-dependent AKT2 activation. Expression of point-mutated CBL4C3S cRNAs that result in myristoylated but nonpalmitoylated CBL4 protein does not evoke AKT2 currents. Currents recorded in oocytes injected with different mixes of cRNAs were normalized to the mean current value at $-160 \mathrm{mV}$ in oocytes injected with AKT2 cRNA only. Data are displayed as mean \pm SE ( $n=$ number of tested oocytes) for the following injected mixes of cRNAs: $A K T 2(n=25), A K T 2+C I P K 6+C B L 4(n$ $=23$ ) and $A K T 2+C I P K 6+C B L 4 C 3 S(n=18)$. (C and D) CBL4 missing N-terminal lipid modifications fail to induce the translocation of AKT2/CIPK6 complexes. Microscopic analysis of the median cellular plane of $N$. benthamiana epidermal cells transiently expressing the plasmid combinations indicated at the left. A white arrow marks the region and direction in which the distribution of fluorescence intensities was determined. (C) Co-expression of CBL4G2A-SCFP and (D) CBL4C3S-SCFP lead to a retention of the AKT2/CIPK6 BiFC signal (green) at the ER, which is clearly distinct from the PM-marker CBL1n-OFP (red). 
phosphorylation activity. Moreover, injecting BAPTA or replacing the wild-type CBL4 protein by its $\mathrm{EF}$ hand mutated protein version CBL4 $\triangle \mathrm{EF}$ (which cannot bind $\mathrm{Ca}^{2+}$ ) abolished activation of AKT2 currents in oocytes (Figure 5D) and AKT2 ER-to-PM translocation in BiFC experiments (Figure 5E, Supplementary information, Figure S5B). These results clearly establish the $\mathrm{Ca}^{2+}$ dependence of CIPK6C-mediated AKT2 modulation. However, they currently do not allow us to distinguish whether this $\mathrm{Ca}^{2+}$ dependence just reflects a "constitutive" structural requirement that may contribute to CBL4-CIPK6 interaction in forming an ER-to-PM escort complex or, instead, could allow specific $\mathrm{Ca}^{2+}$-dependent control of the traffic of AKT2 channels to the cell surface.

Dual lipid modifications of CBL4 by myristoylation and palmitoylation are required for AKT2 activation and $P M$ targeting

A recent investigation of CBL1 established the importance of myristoylation and palmitoylation of this $\mathrm{Ca}^{2+}$ sensor for its proper function [12]. The crucial importance of both lipid modifications is due to their function as signals in cytoplasm-to-ER and ER-to-PM trafficking of this $\mathrm{Ca}^{2+}$ sensor protein. Modification of CBL1 by palmitoylation was identified as the key in targeting CBL1/CIPK1 complexes by a novel BFA- and Sar1independent sorting pathway to the PM [12]. This work also established the myristoylation at the $\mathrm{G} 2$ position of all four PM-localized CBL proteins, including CBL4, and reported the conservation of the palmitoylated $\mathrm{C} 3$ position in all four PM-localized CBL proteins, including CBL4. Therefore, a similar PM-targeting mechanism that relies on dual lipid modifications for these proteins was proposed [12]. In order to address the importance of dual lipid modifications for CBL4 function in AKT2 regulation and targeting, we generated non-myristoylatable G2A version and non-palmitoylatable C3S version of CBL4. When wild-type CBL4 was replaced by the nonmyristoylatable CBL4G2A in TEVC analysis in oocytes, we observed a complete abolishment of AKT2 activation (Figure 6A). Likewise, injection of the non-palmitoylatable CBL4C3S together with AKT2 and CIPK6 did not result in any detectable activation of AKT2-dependent currents (Figure 6B). In order to investigate the effect of either myristoylation or palmitoylation of CBL4 on AKT2 targeting in planta we co-expressed either the CBL4G2A-SCFP or CBL4C3S-SCFP fusion protein together with AKT2-YC, YN-CIPK6 and the CBL1n PMmarker in $N$. benthamiana cells. In contrast to our previous experiments in which co-expression of wild-type CBL4-SCFP led to an efficient accumulation of AKT2 at the PM (Figure 3C) neither the expression of CBL4G2A-
SCFP nor the expression of CBL4C3S-SCFP was able to bring about ER-to-PM targeting of AKT2 (Figure 6C and $6 \mathrm{D}$ ). These findings indicate that efficient PM targeting of AKT2 requires dual lipid modifications of CBL4 by myristoylation and palmitoylation, and suggest that this translocation occurs via the novel BFA- and Sar1independent targeting pathway that was established for CBL1.

\section{Discussion}

In this study we provide new insights into the regulation of $\mathrm{K}^{+}$channel activity. Previous studies have revealed the first important features of plant $\mathrm{K}^{+}$channel regulation by CBLs and CIPKs. CBL1(CBL9)/ CIPK23 complexes have been shown to mediate $\mathrm{Ca}^{2+}$ and phosphorylation-dependent activation of the AKT1 channel, which is targeted to the PM independently of CBL1/CIPK23 [1-3]. Our study extends the range of CBL-CIPK-regulated $\mathrm{K}^{+}$channels, in that it identifies the AKT2 $\mathrm{K}^{+}$channel as a target of this $\mathrm{Ca}^{2+}$-decoding signaling network. The modulation of AKT2 activity is brought about by the combined function of CBL4 and CIPK6. In agreement with the function of CBL4 and CIPK6 in modulating AKT2 activity, loss-of-function mutants of either CBL4, CIPK6 or AKT2 exhibit a developmental phenotype under short-day cultivation conditions that is manifested by delayed development and flowering in these mutants as compared to wild type. Our results extend the range of physiological functions of this $\mathrm{Ca}^{2+}$ sensor (also designated SOS3) that originally has been described as a critical component of salt-stress signaling [26]. This study also extends the known functions of CIPK6 for which a previous analysis of a T-DNA knock-down mutant of Arabidopsis that accumulates only $50 \%$ of the wild-type level of CIPK 6 mRNA revealed a role of this kinase in salt tolerance and defect in auxin distribution [29]. Remarkably, the results presented here do not support a regulation of AKT2 by CIPK6dependent phosphorylation. Instead, our data suggest an ER-to-PM translocation step as a critical process of AKT2 activation that is dependent on interaction of AKT2 with the CBL4/CIPK6 complex. This adds a new layer of complexity to the mechanisms of plant $\mathrm{K}^{+}$channel regulation by CBL/CIPK complexes. Stimulation of AKT2 current by CBL4/CIPK6 appears to be strictly $\mathrm{Ca}^{2+}$-dependent as it is abolished by both chelating $\mathrm{Ca}^{2+}$ ions by BAPTA application and impairing $\mathrm{Ca}^{2+}$ binding of CBL4 by EF hand mutation. In fluorescence imaging studies the CBL4 EF hand mutant failed to promote PM translocation of the AKT2/CIPK6 complex, suggesting a strict $\mathrm{Ca}^{2+}$ dependence of this targeting pathway. How- 
ever, we cannot currently distinguish whether this $\mathrm{Ca}^{2+}$ dependence of channel translocation represents a constitutive requirement of this process or, instead, allows a $\mathrm{Ca}^{2+}$-dependent regulation of channel trafficking.

In our experiments an artificial truncated CIPK6 protein that encompassed solely the $\mathrm{C}$-terminal regulatory domain and completely lacked the $\mathrm{N}$-terminal kinase domain was able to interact with CBL4 and AKT2, and was fully sufficient to activate AKT2 in oocytes and to enhance AKT2 translocation to the PM in plant cells in a strictly $\mathrm{Ca}^{2+}$ dependent manner. In sharp contrast, the Nterminal domain of CIPK6, which possesses the kinase activity, was not able to bring about any of these effects. Together with our inability to detect any phosphorylation activity of CIPK6 toward AKT2 in vitro, these findings strongly argue for a crucial role of phosphorylationindependent but $\mathrm{Ca}^{2+}$-dependent regulation of AKT2 by CIPK6. This regulation of AKT2 is brought about by the combined action of CBL4 that renders this process $\mathrm{Ca}^{2+}$ dependent and by CIPK6 that fulfills a scaffolding function interconnecting CBL4 with AKT2.

Many characterized channels are retained in the ER, and require association of escort proteins or oligomerization to mask their ER-retention signal and allow them to traffic beyond the ER [19]. Alternatively, autonomous export from the ER can be conveyed by a triacidic motif within the $\mathrm{C}$-terminal domain of $\mathrm{K}^{+}$channels, as exemplified by the targeting mechanism of the Arabidopsis $\mathrm{K}^{+}$channel, KAT1 [21]. However, such an ER export motif appears to be absent in AKT2 [30], a finding that is in agreement with our observation of preferential ER accumulation of homomeric AKT2 channels in $N$. benthamiana cells. Previous studies have identified EF hand-containing KChIP $\mathrm{Ca}^{2+}$ sensor proteins in animals as integral regulatory components of $\mathrm{Kv} 4 \mathrm{~K}^{+}$channels that, by masking a hydrophobic N-terminal domain of the channel, mediate $\mathrm{Ca}^{2+}$-dependent PM translocation of these channels [20, 31, 32]. In the case of Kv4.2, this channel can traffic to the Golgi apparatus by itself. Here, the N-terminal domain of KV4.2, which determines the intracellular retention of the Kv4.2 subunits when they are expressed alone [20, 32, 33], interacts with KChIP1 resulting in efficient $\mathrm{Ca}^{2+}$-dependent $\mathrm{PM}$ translocation of the $\mathrm{K}^{+}$channel $/ \mathrm{Ca}^{2+}$ sensor complex [20]. Our study has revealed a novel mechanism of $\mathrm{Ca}^{2+}$-dependent $\mathrm{K}^{+}$channel regulation in that a $\mathrm{Ca}^{2+}$ sensor-kinase module interdependently regulates the activity of the AKT2 channel. We suggest that the regulatory domain of CIPK6 provides an anchoring module that by $\mathrm{Ca}^{2+}$-dependent interaction with CBL4, which functions as an escort protein, mediates efficient ER-to-PM targeting of the $\mathrm{K}^{+}$channel by a yet-to-be explored trafficking pathway. This model implies that AKT2 due to its membrane-spanning domains would reside in the ER membrane and would get in contact with the CBL4/CIPK6 complex in which the $\mathrm{Ca}^{2+}$ sensor moiety is already myristoylated but not yet palmitoylated. Further palmitoylation of CBL4 would mediate translocation of the ternary complex to the PM. Remarkably, in the case of the Kv4.2 channel, Golgi-toPM membrane translocation requires single lipid modification by myristoylation of its interacting $\mathrm{KChIP} 1 \mathrm{Ca}^{2+}$ sensor subunit [20]. Our work provides a first example in which dual lipid modifications of a $\mathrm{Ca}^{2+}$ sensor by both myristoylation and palmitoylation are necessary for efficient PM targeting of a $\mathrm{K}^{+}$channel. Hasdemir et al. [20] reported that KChIP-dependent trafficking of the Kv4.2 channel was Sar1(H79G) independent, indicating that ER-to-Golgi transport was independent of COPII vesicles. However, our previous work revealed that transport of CBL1/CIPK1 complexes is distinguished from the trafficking of $\mathrm{KChIP} / \mathrm{Kv} 4.2$ complexes by their opposing dependence on COPI vesicles [12]. While blocking of ARF GTPase function interferes with KChIP/ Kv4.2 exocytic transport, such treatment does not affect CBL1/CIPK1 trafficking [12]. Considering the structural conservation of the lipid modification sites in CBL1 and CBL4 and their common requirement of dual lipid modifications for efficient PM targeting, it is tempting to speculate that such a novel BFA- and Sar1-independent targeting pathway mediates targeting of CBL4/CIPK6/ AKT2 ternary complexes. In summary, our findings add important new facets to the complex array of cellular processes regulating ion channel activity.

\section{Materials and Methods}

General methods, construct generation, plant cultivation and phenotype analyses, and yeast two-hybrid studies

Molecular biology methods were performed according to standard procedures. A list of primers used in this work is provided in the Supplementary information, Table S1. In general, for construct generation the respective protein encoding regions of the CBLs and CIPKs were amplified by PCR and introduced into the pGPTVII backbone vectors [34] for localization analyses, into pSPYNE and pSPYCE vectors [35, 36] for BiFC assays and for expression in oocytes cDNAs were cloned into modified pGEMHE vectors [37]. Forward primers with inserted point mutations were used to generate cDNAs encoding the CBL4G2A and CBL4C3S mutants. The identity of all plasmid constructs generated in this study was verified by sequencing. A. thaliana (ecotypes Wassilewskija and Columbia) plants that were used in this work were grown on soil. In this study cbl4 and cipk6 T-DNA insertion lines from the GABI collection were investigated (cbl4: GABI 015F02; cipk6: GABI 448C12). The T-DNA insertions in both mutants were localized by PCR by using T-DNA border-specific and cipk6 and cbl4 gene-specific primers. PCR products for both borders were sequenced. For transcript analysis $1 \mu \mathrm{g}$ of total RNA was 
used for cDNA synthesis and RT-PCR was performed with genespecific primers. Segregation analysis of progenies of heterozygous mutant lines revealed a 3:1 ratio, confirming single T-DNA insertions. To further corroborate the mutant status of the $c b l 4$ and cipk6 lines, salt stress assays were performed as previously described [10], which confirmed the previously reported salt-sensitive phenotypes of published sos3 (here designated as cbl4) lossof-function mutants [23] and published cipk6 mutant alleles [29]. Complementation lines of akt2-1, cipk6 and cbl4 were generated through Agrobacterium-mediated transformation [38]. AKT2 and CIPK6 were expressed under endogenous promoters, CBL4 under control of the MAS promoter.

In $12 \mathrm{~h} / 12 \mathrm{~h}$ photo regime, plants were grown in a growth chamber at $60 \%$ relative humidity, at $20{ }^{\circ} \mathrm{C}$ during the day with a photon flux density of $100 \mu \mathrm{E} \mathrm{m}^{-2} \mathrm{~s}^{-1}$ (Philips TLD58W840) and at $16{ }^{\circ} \mathrm{C}$ during the night. Phenotype analyses were carried out in the middle of the day period. Leaf numbers were monitored once a week starting from 2 weeks after sowing. Main inflorescence stalk height was measured every fourth day starting from stalk emergence. Statistically significant difference between data sets (marked by asterisks above the corresponding bars) was checked by Student's $t$-test ( $95 \%$ threshold). $N$. benthamiana plants were cultivated in a green house under a $12 \mathrm{~h}$ light/12 h dark cycle with $60 \%$ atmospheric humidity at $20{ }^{\circ} \mathrm{C}$. Yeast two-hybrid analyses were performed as described previously $[6,39]$.

\section{Expression in Xenopus oocytes and electrophysiological re- cordings}

Oocytes were obtained and stored as described previously [17]. In vitro transcriptions were performed using the mMESSAGE mMACHINE kit (Ambion) following the manufacturer's instructions. Oocytes were injected with a final volume of $20 \mathrm{nl}$ of various cRNA combination using a 10-15 $\mu \mathrm{m}$ tip diameter micro-pipette. Injections were performed using either $8 \mathrm{ng}$ of Shaker cRNA or, for co-injection experiments, a mix of $8 \mathrm{ng}$ of Shaker cRNA and $6 \mathrm{ng}$ of both CIPK and CBL cRNAs. All experiments were performed at room temperature $\left(20-22{ }^{\circ} \mathrm{C}\right)$. Whole-cell currents were recorded 3-4 days after the injection of oocytes as described previously [17] using the TEVC technique. Voltage-dependent activation of Shaker channels was recorded using a pulse protocol starting from a holding potential of $-40 \mathrm{mV}$, pulses were applied to various test voltages as indicated in legend to Figure 1D. The bath solution contained $100 \mathrm{mM} \mathrm{KCl}, 1 \mathrm{mM} \mathrm{CaCl}_{2}, 1.5 \mathrm{mM} \mathrm{MgCl}_{2}$ and $10 \mathrm{mM}$ HEPES-Tris ( $\mathrm{pH} 7.5$ ). BAPTA injections (50 $\mathrm{nl}$ of $50 \mathrm{mM}$ BAPTA) were performed using a $10-15 \mu \mathrm{m}$ tip diameter micropipette.

Patch-clamp experiments (oocyte-attached configuration) were performed as described previously [14] using bath and pipette solutions identical to above-described bath solution.

pClamp software version 9.0 (Axon instruments), Sigmaplot software (Jandel Scientific) and WINASCD software (G Droogmans, Laboratory of Physiology, Leuven, Belgium) were used to perform voltage-clamp protocol application, data acquisition and data analysis.

Confidence intervals represent SD or SE as indicated in legend to figures. Statistically significant difference between data sets (marked by asterisks above the corresponding bars) was checked by Student's $t$-test (95\% threshold).
In vitro translation, protein purification and in vitro phosphorylation assays

The pIVEX 1.3 WG plasmid (Roche) was used as the template for site-directed mutagenesis PCR to generate the pIVEX WG StrepII vector that expresses N-terminally StrepII-tagged proteins. The respective cDNAs were amplified by PCR and sub-cloned into the pIVEX WG StrepII vector. For in vitro transcription/translation using RTS 500 Wheat Germ CECF Kit (Roche), $60 \mu \mathrm{g}$ of pIVEX WG StrepII constructs were used. Each translation reaction was incubated with Strep-Tactin Macroprep (IBA) for 30 min at $4{ }^{\circ} \mathrm{C}$ and the mixture was transferred to a microspin-column (Bio-Rad) for gravity-flow procedures. The column was washed with wash buffer (100 mM Tris/HCl, pH8, $150 \mathrm{mM} \mathrm{NaCl).} \mathrm{StrepII-tagged}$ protein was eluted with elution buffer (wash buffer containing 2.5 $\mathrm{mM}$ Desthiobiotin). The concentrations of purified proteins were determined by comparison with Coomassie-stained BSA in SDSPAGE.

For in vitro phosphorylation assays, approximately $200 \mathrm{ng}$ of each purified protein was incubated for $30 \mathrm{~min}$ at $30{ }^{\circ} \mathrm{C}$ in $24 \mu 1$ reaction mixes, $16 \mu \mathrm{g}$ of BSA, $66.7 \mathrm{mM}$ Tris/ $\mathrm{HCl}(\mathrm{pH} 8.0), 100$ $\mathrm{mM} \mathrm{NaCl}_{2}, 5 \mathrm{mM} \mathrm{MnSO}_{4}, 0.5 \mathrm{mM} \mathrm{CaCl}_{2}, 2 \mathrm{mM}$ dithiothreitol, 0.5 $\mu \mathrm{M}$ ATP and $4 \mu \mathrm{Ci}$ [gamma $-{ }^{32} \mathrm{P}$ ] ATP. The reaction was stopped by adding $1 \mu \mathrm{l}$ of $0.5 \mathrm{M}$ EDTA, and the mixtures were separated by SDS-PAGE. The gel was stained with $\mathrm{CBB}$ and exposed to an $\mathrm{X}$-ray film.

\section{Fluorescence microscopy}

Transient expression of XFP-fusion proteins in $N$. benthamiana and BiFC were previously described $[12,35,36]$. For co-expression of the investigated proteins and co-localisation with PM and ER-markers, pGPTVII vectors containing AKT2-Venus, CBL1nOFP, OFP-HDEL, CBL4-OFP and CBL4-SCFP under control of the $35 \mathrm{~S}$ promoter or MAS promoter in case of CBL1n-OFP were transformed into agrobacteria and co-infiltrated at a OD of 0.5 , except for the HDEL-OFP bearing strain, which was infiltrated at an $\mathrm{OD}$ of 0.1 . Fluorescence emissions were determined 3 days after infiltration for CIPK6-CBL4 BiFC combinations and 4 days after infiltration in experiments containing CIPK6-AKT2 BiFC combinations using a Leica DMIRE2 with TCS SP2 laser scanner setup with a HCX PL APO $63 \times 1.2$ water objective (Leica Microsystems $\mathrm{GmbH}$ ). For YFP and OFP excitation a 488- or 514-nm (Ar/ $\mathrm{Kr})$ laser and 543- or 594-nm $(\mathrm{He} / \mathrm{Ne})$ laser was used, respectively, and emission was recorded at 518-560 nm (YFP) and 605-640 nm (OFP). SCFP fluorescence was generally recorded separately of YFP fluorescence with a 405-nm diode laser and detected at 460$480 \mathrm{~nm}$. Fluorescence intensity scans were performed as described [12] using the quantification tool of the Leica confocal software application (version 2.61). Microscopy was done at room temperature with leaf discs immersed in water.

\section{Acknowledgments}

We are grateful to Leonie Steinhorst for providing the cbl4 mutant line, to Whitney Robertson for providing the akt2-1 mutant line, to Dr Oliver Batistič for providing CBL4G2A and CBL4C3S constructs. We also thank Dr Dietmar Geiger for providing the pGEMKN plasmid. FP and CC-F were supported by grants from CNRS and ANR Genoplante, respectively. This work was supported by grants from the DFG to ID (Heisenberg-fellowship and 
DFG DR 430/5-1 and 5-2) and to JK (SFB 629/B9 and AFGN Ku931/7-1), from the HFSP to JK (RGP033/2006-C) and from the ANR Genoplante to CC-F, BL and J-BT (ANR-06-GPLA-012).

\section{References}

$1 \mathrm{Xu}$ J, Li HD, Chen LQ et al. A protein kinase, interacting with two calcineurin B-like proteins, regulates $\mathrm{K}^{+}$transporter AKT1 in Arabidopsis. Cell 2006; 125:1347-1360.

2 Lee SC, Lan WZ, Kim BG et al. A protein phosphorylation/ dephosphorylation network regulates a plant potassium channel. Proc Natl Acad Sci USA 2007; 104:15959-15964.

3 Cheong YH, Pandey GK, Grant JJ, et al. Two calcineurin Blike calcium sensors, interacting with protein kinase CIPK23, regulate leaf transpiration and root potassium uptake in Arabidopsis. Plant J 2007; 52:223-239.

4 Batistič O, Kudla J. Plant calcineurin B-like proteins and their interacting protein kinases. Biochim Biophys Acta 2009; 1793:985-992.

5 Weinl S, Kudla J. The CBL-CIPK $\mathrm{Ca}^{2+}$-decoding signaling network: function and perspectives. New Phytol 2009; 184:517-528.

6 Albrecht V, Ritz O, Linder S, Harter K, Kudla J. The NAF domain defines a novel protein-protein interaction module conserved in $\mathrm{Ca}^{2+}$-regulated kinases. EMBO J 2001; 20:10511063.

7 Batistič O, Kudla J. Integration and channeling of calcium signaling through the CBL calcium sensor/CIPK protein kinase network. Planta 2004; 219:915-924.

8 Kudla J, Batistič O, Hashimoto K. Calcium signals: the lead currency of plant information processing. Plant Cell 2010; 22:541-563.

9 Albrecht V, Weinl S, Blazevic D, et al. The calcium sensor CBL1 integrates plant responses to abiotic stresses. Plant $J$ 2003; 36:457-470.

10 D'Angelo C, Weinl S, Batistič O, et al. Alternative complex formation of the $\mathrm{Ca}^{2+}$-regulated protein kinase CIPK1 controls abscisic acid-dependent and independent stress responses in Arabidopsis. Plant J 2006; 48:857-872.

11 Kim BG, Waadt R, Cheong YH, et al. The calcium sensor CBL10 mediates salt tolerance by regulating ion homeostasis in Arabidopsis. Plant J 2007; 52:473-484.

12 Batistič O, Sorek N, Schultke S, Yalovsky S, Kudla J. Dual fatty acyl modification determines the localization and plasma membrane targeting of CBL/CIPK $\mathrm{Ca}^{2+}$ signaling complexes in Arabidopsis. Plant Cell 2008; 20:1346-1362.

13 Marten I, Hoth S, Deeken R, et al. AKT3, a phloem-localized $\mathrm{K}^{+}$channel, is blocked by protons. Proc Natl Acad Sci USA 1999; 96:7581-7586.

14 Lacombe B, Pilot G, Michard E, et al. A shaker-like $\mathrm{K}^{+}$channel with weak rectification is expressed in both source and sink phloem tissues of Arabidopsis. Plant Cell 2000; 12:837851.

15 Dreyer I, Michard E, Lacombe B, Thibaud JB. A plant Shaker-like $\mathrm{K}^{+}$channel switches between two distinct gating modes resulting in either inward-rectifying or "leak" current. FEBS Lett 2001; 505:233-239.

16 Cherel I, Michard E, Platet N, et al. Physical and functional interaction of the Arabidopsis $\mathrm{K}^{+}$channel AKT2 and phos- phatase AtPP2CA. Plant Cell 2002; 14:1133-1146.

17 Michard E, Dreyer I, Lacombe B, Sentenac H, Thibaud JB. Inward rectification of the AKT2 channel abolished by voltage-dependent phosphorylation. Plant J 2005; 44:783-797.

18 Michard E, Lacombe B, Poree F, et al. A unique voltage sensor sensitizes the potassium channel AKT2 to phosphoregulation. J Gen Physiol 2005; 126:605-617.

19 Ma D, Jan LY. ER transport signals and trafficking of potassium channels and receptors. Curr Opin Neurobiol 2002; 12:287-292.

20 Hasdemir B, Fitzgerald DJ, Prior IA, Tepikin AV, Burgoyne RD. Traffic of Kv4 $\mathrm{K}^{+}$channels mediated by KChIP1 is via a novel post-ER vesicular pathway. J Cell Biol 2005; 171:459469.

21 Mikosch M, Kaberich K, Homann U. ER export of KAT1 is correlated to the number of acidic residues within a triacidic motif. Traffic 2009; 10:1481-1487.

22 Xicluna J, Lacombe B, Dreyer I, et al. Increased functional diversity of plant $\mathrm{K}^{+}$channels by preferential heteromerization of the shaker-like subunits AKT2 and KAT2. J Biol Chem 2007; 282:486-494.

23 Ishitani M, Liu J, Halfter U, et al. SOS3 function in plant salt tolerance requires N-myristoylation and calcium binding. Plant Cell 2000; 12:1667-1678.

24 Dennison KL, Robertson WR, Lewis BD, et al. Functions of AKT1 and AKT2 potassium channels determined by studies of single and double mutants of Arabidopsis. Plant Physiol 2001; 127:1012-1019.

25 Deeken R, Geiger D, Fromm J, et al. Loss of the AKT2/3 potassium channel affects sugar loading into the phloem of Arabidopsis. Planta 2002; 216:334-344.

26 Quintero FJ, Ohta M, Shi H, Zhu JK, Pardo JM. Reconstitution in yeast of the Arabidopsis SOS signaling pathway for $\mathrm{Na}^{+}$homeostasis. Proc Natl Acad Sci USA 2002; 99:90619066.

27 Fujii H, Zhu JK. An autophosphorylation site of the protein kinase SOS2 is important for salt tolerance in Arabidopsis. Mol Plant 2009; 2:183-190.

28 Mahajan S, Sopory SK, Tuteja N. Cloning and characterization of CBL-CIPK signalling components from a legume (Pisum sativum). FEBS J 2006; 273:907-925.

29 Tripathi V, Parasuraman B, Laxmi A, Chattopadhyay D. CIPK6, a CBL-interacting protein kinase is required for development and salt tolerance in plants. Plant $J$ 2009; 58:778790.

30 Mikosch M, Homann U. How do ER export motifs work on ion channel trafficking? Curr Opin Plant Biol 2009; 12:685689.

31 An WF, Bowlby MR, Betty M, et al. Modulation of A-type potassium channels by a family of calcium sensors. Nature 2000; 403 (6769):553-556.

32 Shibata R, Misonou H, Campomanes CR, et al. A fundamental role for KChIPs in determining the molecular properties and trafficking of Kv4.2 potassium channels. J Biol Chem 2003; 278:36445-36454.

33 Bahring R, Dannenberg J, Peters HC, et al. Conserved Kv4 $\mathrm{N}$-terminal domain critical for effects of $\mathrm{Kv}$ channel-interacting protein 2.2 on channel expression and gating. J Biol Chem 2001; 276:23888-23894. 
34 Shi J, Kim KN, Ritz O, et al. Novel protein kinases associated with calcineurin B-like calcium sensors in Arabidopsis. Plant Cell 1999; 11:2393-2405.

35 Walter M, Chaban C, Schutze K, et al. Visualization of protein interactions in living plant cells using bimolecular fluorescence complementation. Plant J 2004; 40:428-438.

36 Waadt R, Schmidt LK, Lohse M, et al. Multicolor bimolecular fluorescence complementation reveals simultaneous formation of alternative CBL/CIPK complexes in planta. Plant $J$ 2008; 56:505-516.
37 Liman ER, Tytgat J, Hess P. Subunit stoichiometry of a mammalian $\mathrm{K}^{+}$channel determined by construction of multimeric cDNAs. Neuron 1992; 9:861-871.

38 Clough SJ, Bent AF. Floral dip: a simplified method for Agrobacterium-mediated transformation of Arabidopsis thaliana. Plant J 1998; 16:735-743.

39 Kolukisaoglu Ü, Weinl S, Blazevic D, Batistič O, Kudla J. Calcium sensors and their interacting protein kinases: genomics of the Arabidopsis and rice CBL-CIPK signaling networks. Plant Physiol 2004; 134:43-58.

(Supplementary information is linked to the online version of the paper on the Cell Research website.) 